\title{
A New Three-Dimensional Chaotic System with Constant Exponent Spectrum: Analysis, Synchronization and Circuit Implementation
}

\author{
Jian Jiang ${ }^{*}$, Qing-Qing $\mathrm{Wu}^{2}$ \\ ${ }^{1}$ Institute of software, School of Statistics and Applied Mathematics, Fudan University, Shanghai 201203, \\ China. \\ 2 Jiangsu Zhongtian technology Software Technology Co., Ltd., Nantong 226000, China. \\ * Corresponding author. Tel.: 18206272311; email: qingqingwu1989@163.com \\ Manuscript submitted February 2, 2016; accepted March 20, 2016. \\ doi: $10.17706 /$ jsw.11.5.494-511
}

\begin{abstract}
A new chaotic system with constant Lyapunov exponent spectrum is presented, two system parameters of which remain constant exponent characteristics. The basic dynamic properties of the new system are analyzed by Poincar section, Lyapunov exponent and dimension and the signal power spectrum. Based on the Lyapunov exponent spectrum, bifurcation diagrams and state variable amplitude with respect to system parameters show that the new system has two parameters with globally nonlinear modulation characteristic. Based on the Lyapunov stability theory and the improvement of the traditional method, through constructing a Lyapunov function with containing a positive constant that the complete synchronization with single controllers are achieved by means of nonlinear active feedback, adaptive, backstepping and passive control theory, respectively. And the designed single controllers are verified via numerical simulation. Because of single controller, synchronous switch circuit consists of four synchronization schemes is implemented, which further validate the correctness and practicability of the single controllers.
\end{abstract}

Key words: Chaotic system, Lyapunov exponent, synchronization, single controller, circuit implementation.

\section{Introduction}

Chaotic system is discovered by America meteorologist Lorenz in the last century 60's [1]. Subsequently, the research and application of chaos theory in many fields has attracted much attention. Until the early ninety's Pecoro and Carroll firstly achieved synchronization of chaotic system and implementation of synchronous circuit [2], [3] by using variable substitution method. Research on chaos synchronization has been found extensive application prospect in secure communication, bioengineering, image encryption and other disciplines [4]-[6], so more and more scholars devoted to the research of chaos synchronization. Studies on chaos are mainly including the dynamic characteristics of the system analysis, synchronization control of chaotic system, and circuit design and physically implemented [7]-[9].

With the development of chaos theory and computer technology, various kinds of new chaotic systems have been found in [10]-[13]. To the best of our knowledge, the reports and variety about a chaotic system with constant Lyapunov exponent spectrum are rare. Li Chunbiao et al proposed several kinds of chaotic systems with constant Lyapunov exponent spectrum, which have common characteristics that strange attractor is generated from the absolute terms[14]-[16]. Zhou Xiaoyong presented a new chaotic system 
with constant Lyapunov exponent spectrum and investigated the functional relationship between the constant exponent parameters and the amplitude of the signal of the system[17]. Because of the chaotic system with constant Lyapunov exponent spectrum has more special dynamic characteristics than the general one, which has great application prospect in the circuit realization and cost control in industrial application, so discovery and study of chaotic system with constant LE spectrum become another one research hotspot of chaos theory. Chaotic synchronization achieved fruitful results in recent years. Hybrid phase synchronization for a three-dimensional chaotic system was discussed with active feedback control method in literature [18]. References therein [19] achieved synchronous control of Chua's circuit based on the symbol function. Synchronization of chaotic systems with unknown parameters was investigated by utilizing adaptive method [20], and passive synchronization control, backstepping method [21]-[23], and so on. However, during the above employed approaches, controllers are designed more than one, which will be difficult in industrial implementation and cost control in the future. To the best of our knowledge, a synchronous circuit implemented contains adaptive synchronization, active synchronization, backsteeping synchronization and passive synchronization has never been reported ever. And the synchronous performance of single controller or multi-controller is the same. Therefore decrease the number and simplify the structure of synchronization controllers are the research objectives of chaos synchronization theory.

This paper presents a new three-dimensional autonomous chaotic system with constant Lyapunov exponent spectrum of double parameters contains five variable parameters, and the dynamic characteristics of the system is generated through the cross-product terms and a quadratic component. First, the dynamic characteristics of the new system are investigated by means of theoretical derivation, numerical simulation, Lyapunov dimension and exponent, Poincare section, power spectrum, LE spectrum and bifurcation diagram. And the characteristics for amplitude and phase inversion of the parameters with constant exponent are studied. Because of the improvement of the traditional method and the particularity of system structure, based on Lyapunov stability theory, the new system can be successively synchronized with a single controller by means of active control, adaptive control, passive control, backstepping control and so on. The numerical simulation is carried out to verify the validity of the above approaches of synchronization. Due to the single controllers, the switching synchronization can be easily realized via experimental circuit. Finally, the design of synchronous switch circuit was realized including four synchronization schemes and operated through different combination of switches, which further illustrate the practicability of synchronization.

This paper is organized as follows. In Section 2, the basic dynamic characteristics of the system are given. In Section 3, four kinds of synchronization schemes of the system are given, and the correctness of the schemes are proved through Lyapunov stability theorem. In Section 4, the correctness of the designed controllers are proved via the numerical simulation. In Section 5, synchronous switching circuit is designed.

\section{The New System}

In [24], Lü presented a chaotic system, which connects the Lorenz system and Chen system and represents the transition from one to the other. Lü system is described by

$$
\begin{aligned}
& \dot{x}=-a(x-y) \\
& \dot{y}=-x z+c y \\
& \dot{z}=-b z+x y
\end{aligned}
$$

We replace $a x$ and $x z$ with $y^{2}$ and $d z$, respectively. And introducing a system parameter $g$ to the third equation of the above system, a new three-dimensional continuous autonomous system with five 
parameters is obtained as

$$
\begin{aligned}
& \dot{x}=-a\left(x-y^{2}\right) \\
& \dot{y}=-c z+b y \\
& \dot{z}=-d z+g x y
\end{aligned}
$$

where $a, b, c, d, g$ are constant parameters of system, $x, y, z$ are state variables. The system has two strange attractors are shown in Fig. 1 with $a=1, b=2, c=1, d=4, g=1$. The existence of the attractors is illustrated via analysing the basic dynamic properties of the system.
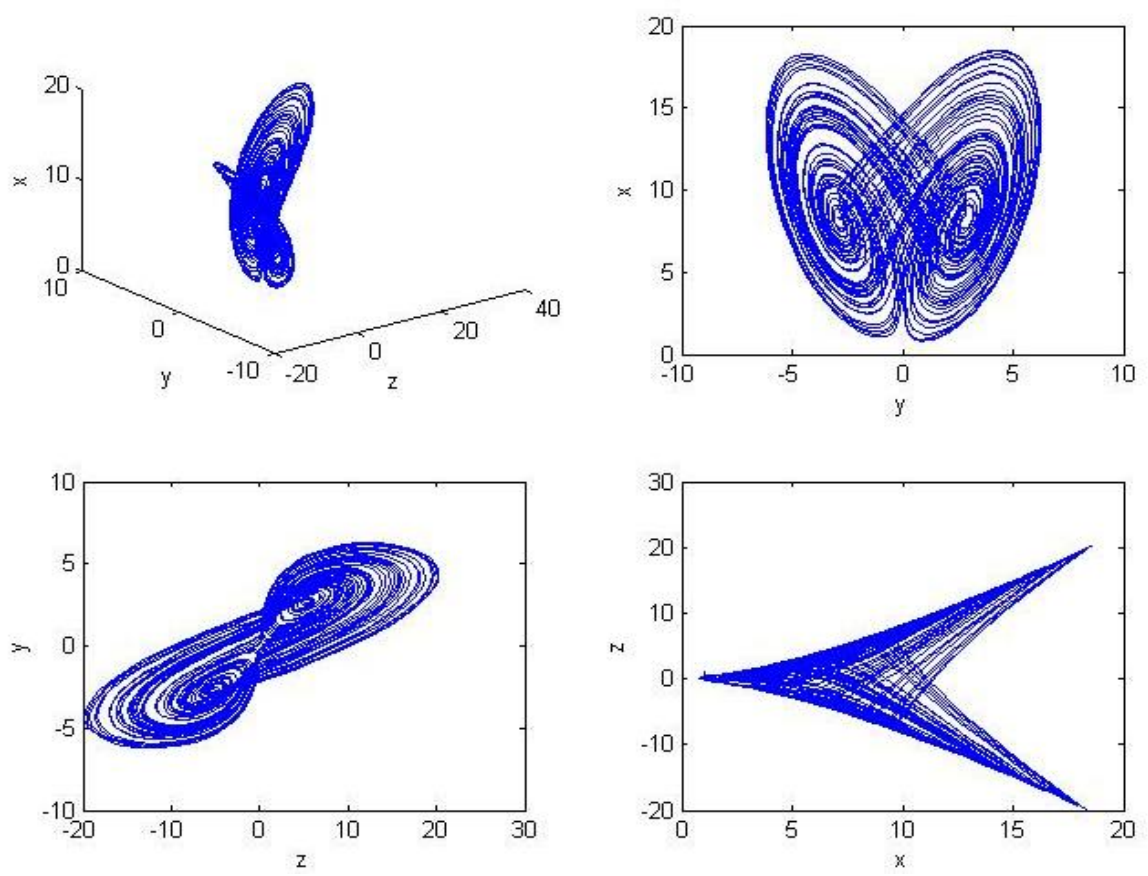

Fig. 1. Attractor of system (1) (a) 3D phase diagram (b) projection $x-y$ plane(c) projection in $z-x$ plane (d) projection in $z-y$ plane.

Remark 1: The literature [13] proposes a three-dimensional chaotic system with multiple attractors in case of different system parameters, which contains only the cross quadratic terms and four system parameters. However, system (1) also has multiple chaotic attractors with selecting the proper system parameters, and which is a system with constant Lyapunov exponent spectrum of double-parameters.

1) Poincaré cross section and the signal power spectrum of the system are shown in Fig.2. The poincaré maps projected on different planes are shown in Fig. 2 (a) (b) (c) where there exist patches of dense points with fractal structure [12] and Fig. 2 (d) (e) (f) are the continuous power spectrum of different system signals which imply the system (1) is a chaotic system.

2) The divergence of system (1) is given by $\Delta V=\frac{\partial \dot{x}}{\partial x}+\frac{\partial \dot{y}}{\partial y}+\frac{\partial \dot{z}}{\partial z}=-a+b-d$, clearly, we have $\Delta V<0$ with $a=1, b=2, d=4$. Therefore, system (1) is dissipative. Dissipative exponential convergence rate is $\mathrm{e}^{-3}[12]$. The volume element of system shrinks to a specific subset of zero volume at an exponential rate $\mathrm{e}^{-3}$ with the time evolution, that is to say the motion of the system can be fixed to a strange attractor. Three equilibria of the system obtained by sloving the equation $\dot{x}=\dot{y}=\dot{z}=0$, which are described respectively as: $s_{1}=(0,0,0), s_{2}=\left(\frac{d b}{c g}, \sqrt{\frac{d b}{g c}}, \sqrt{\frac{d b^{3}}{g c^{3}}}\right), s_{2}=\left(\frac{d b}{c g},-\sqrt{\frac{d b}{g c}},-\sqrt{\frac{d b^{3}}{g c^{3}}}\right)$.The Jacobian 
matrix $J$ obtained by linearing system (1) about equilibrium points is defined as $\boldsymbol{J}=$ $\left[\begin{array}{ccc}-a & 2 a y & 0 \\ 0 & b & -c \\ g y & g x & -d\end{array}\right]_{S_{1}, S_{2}, S_{3}}$. In order to calculate conveniently, substitute $a=1, b=2, c=1, d=4$ and $g=1$ into equilibrium points such that $S_{2}=(8,2 \sqrt{2}, 2 \sqrt{3}), S_{3}=(8,-2 \sqrt{2},-2 \sqrt{3})$. The eigenvalues of $J$ correspond to equilibrium point $S_{1}$ are obtained as $\lambda_{1}=-1, \lambda_{2}=2, \lambda_{3}=-4$, and eigenvalues correspond to equilibrium points $S_{2} S_{3}$ are as $\lambda_{1}=-3.6520, \lambda_{2}=0.3260+2.0676 \mathrm{i}, \lambda_{3}=0.3260-2.0676 \mathrm{i}$. According to the Routh-Hurwitz condition, the equilibrium point is stable only when all of eigenvalues for the Jacobian matrix correspond to the equilibrium points have negative real part, so $S_{1}, S_{2}, S_{3}$ are unstable the system can produce chaotic.

3) Lyapunov exponents are used to measure the exponential rates of divergence and convergence of nearby trajectories, which is an important characteristic to judge the system whether is in a chaotic state or not. The existence of at least one positive Lyapunov exponent implies the system is chaos system.

Three Lyapunov exponents to system (1) were obtained using Jacobi matrix method as: $\lambda_{1}=0.241188$, $\lambda_{2}=0.000925, \lambda_{3}=-3.242113, \lambda_{1}>0$ implies system(1) is chaotic system. The same can be determined according to the Lyapunov dimension:

$$
D_{L}=j+\frac{1}{\left|\lambda_{j+1}\right|} \sum_{i=1}^{j} \lambda_{i}=2+\frac{0.241188+0.000925}{3.242113}=2.074
$$

According to the above computational formula of Lyapunov dimension, Lyapunov dimension is fractal which illustrates system (1) is chaotic system.
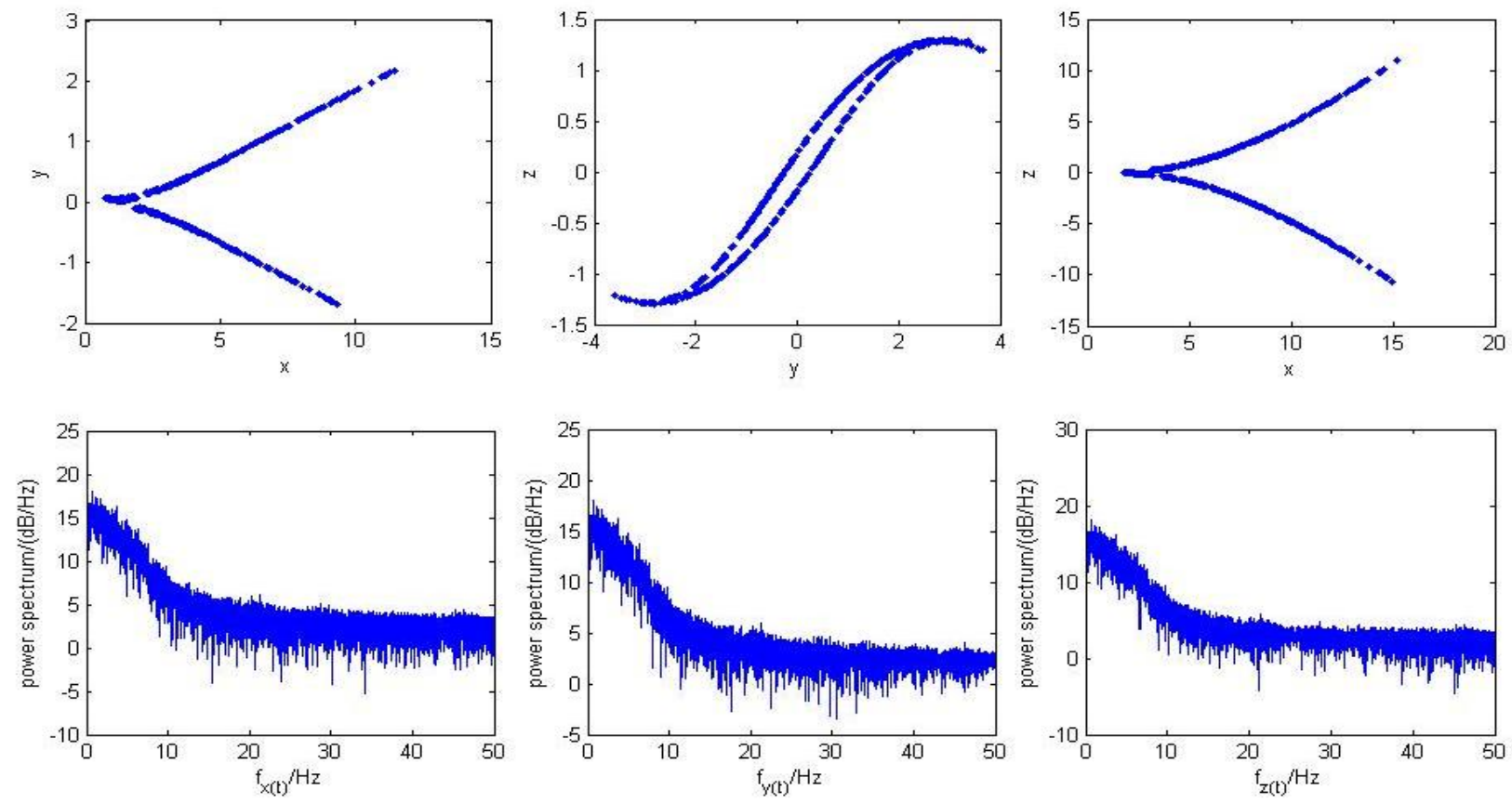

Fig. 2. Poincare section and power spectrum (a) $z=0$ (b) $x=3$ (c) $y=0$ (d) $x(t)$ (e) $y(t)(f) z(t)$.

\section{Constant Exponent Characteristic}

According to the Jacobi matrix $J$ to find out the characteristic polynomial:

$$
f(\lambda)=(a+\lambda)(b-\lambda)(d+\lambda)-2 a \operatorname{cg} y^{2}-(a+\lambda) \operatorname{cg} x
$$


Substituting the three equilibrium points into (2), we get

$$
\begin{gathered}
f(\lambda)_{s 1}=(a+\lambda)(b-\lambda)(d+\lambda) \\
f(\lambda)_{s_{1}, s_{2}}=(a+\lambda)(b-\lambda)(d+\lambda)-(a+\lambda) d b-2 a d b
\end{gathered}
$$

According to (3), (4), eigenvalues of Jacobi matrix corresponding to equilibrium points of the system have nothing to do with the values of system parameters $c$ and $g$, the same as the eigenvalues of $\boldsymbol{J}$ obtained from system (1) linearized at any points, that is to say changes of parameters $c, g$ will not affect the dynamic characteristics of system (1) at the equilibrium point and any points in the phase space. When $a=1, b=2$, $d=4, g=1,1 \leq c \leq 10$, Lyapunov exponents of the system and the bifurcation diagram are shown in Fig. 3 . When $a=1, b=2, c=1, d=4,1 \leq g \leq 10$, Lyapunov exponents of the system and the bifurcation diagram are described in Fig. 4.
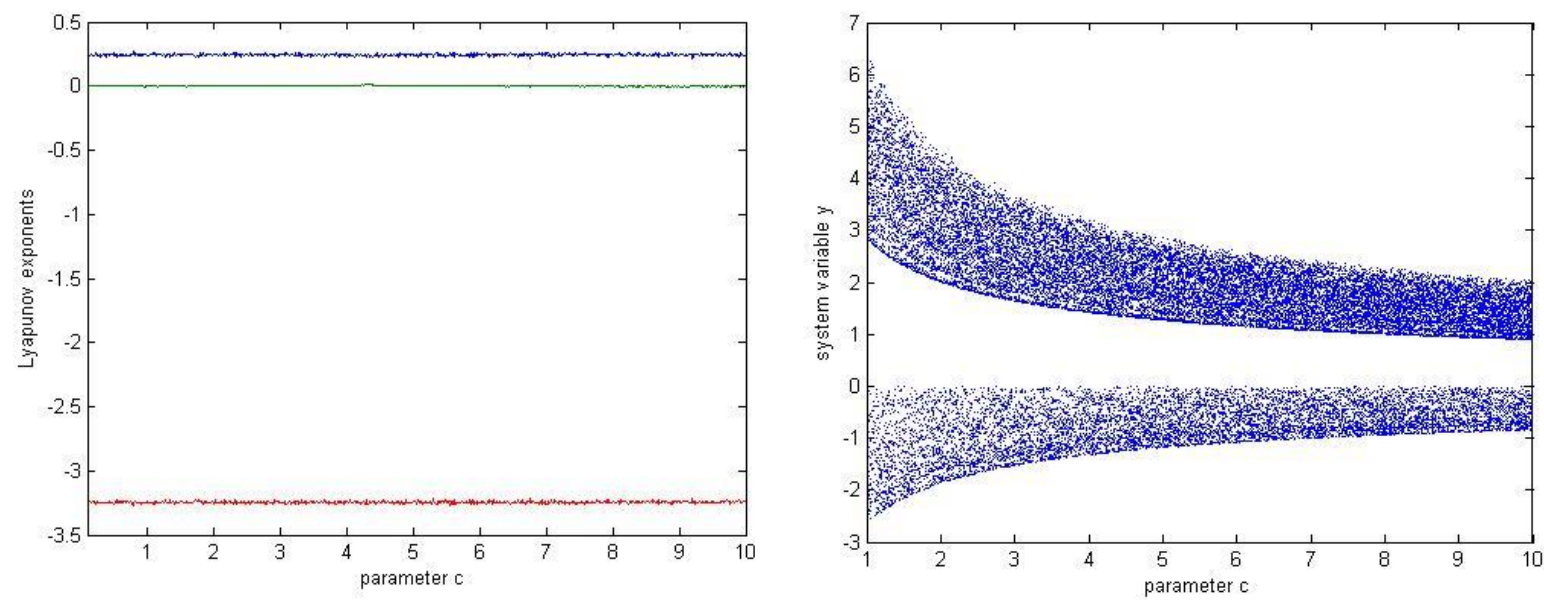

Fig. 3. Lyapunov exponents spectrum and bifurcation diagram (a) Lyapunov exponents spectrum versus $c \in\left[\begin{array}{ll}1 & 10\end{array}\right]$ (b) bifurcation diagram versus $c \in\left[\begin{array}{ll}1 & 10\end{array}\right]$.
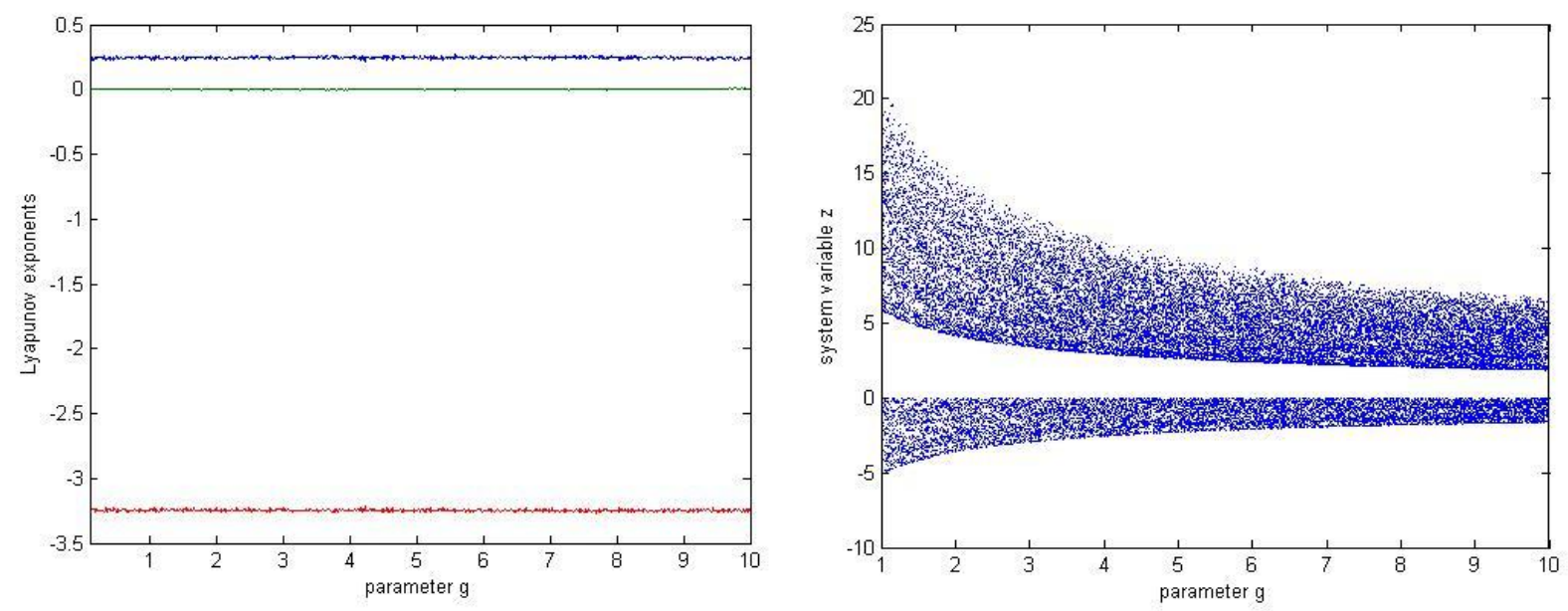

Fig. 4. Lyapunov exponents spectrum and bifurcation diagram (a) Lyapunov exponents spectrum versus $g \in\left[\begin{array}{ll}1 & 10\end{array}\right]$ (b) bifurcation diagram versus $g \in\left[\begin{array}{ll}1 & 10\end{array}\right]$.

Fig. 3(a) and Fig.4 (a) clearly show the value of three Lyapunov exponents unchanged vary with the time that one is positive, for another is close to zero and the last one is negative. Because of the influence of the calculation accuracy, three Lyapunov exponents of the system around a fixed value fluctuate. Three Lyapunov exponents remains $L E_{1}=0.25, L E_{2}=0, L E_{3}=-3.25$ in the case of fixing $a=1, b=2, c=1, d=4, g=1$ and varying $c$ and $g$ between 1 and 10 . 
Only when the system parameters have constant exponent characteristics, the parameters have function of amplitude and phase inversion. Amplitude modulation is divided into local and global amplitude modulation. The modulation of local means a signal in the system changes in linear or non-linear transformation is equivalent to a parameter of the system changes in scale transformation, and the global amplitude modulation is that all signals of the system change in linear or non-linear transformation is equal to a parameter of the system changes in scale transformation, which provide a theoretical basis for communication system of multi parameter modulation.

Theorem 1. System parameters $c$ and $g$ are the global nonlinear modulation parameters, and change with power function relation with the system output signal.

Proof. let $x=k^{2} x^{*}, y=k y^{*}, z=k^{3} z^{*},(k \neq 0)$, system (1) can be rewritten as

$$
\begin{aligned}
& \dot{x}^{*}=-a\left(x^{*}-y^{* 2}\right) \\
& \dot{y}^{*}=-c k^{2} z^{*}+b y^{*} \\
& \dot{z}^{*}=-d z^{*}+g x^{*} y^{*}
\end{aligned}
$$

Accroding to (5), parameter $c$ has global non-linear amplitude modulation character that when the system signals $x, y, z$ in turn into the original $k^{2}, k, k^{3}$ times which is equal to the parameter $c$ becomes 2 times of the original. Therefore, the appropriate changes in amplitude of $c$ can adjust the system signal amplitude. Similarly substitute $x=k^{2} x^{*}, y=k y^{*}, z=k z^{*}$ into the system (1) we can get the relationship between parameter $g$ and the state variables of the system. When $c \in\left[\begin{array}{ll}1 & 10\end{array}\right]$ and $g \in\left[\begin{array}{ll}1 & 10\end{array}\right]$, the maximum and the minimum amplitude of the signal are shown in Fig. 5. As can be seen from Fig. 5, as for the chaotic system with constant Lyapunov exponent spectrum, parameter changes will make the system signal amplitude at any size without changing the characteristics of the system, which greatly expands the scope used in secure communication system. The system signal can be used as carrier signal of any secret signal in theory other than the general chaos system.
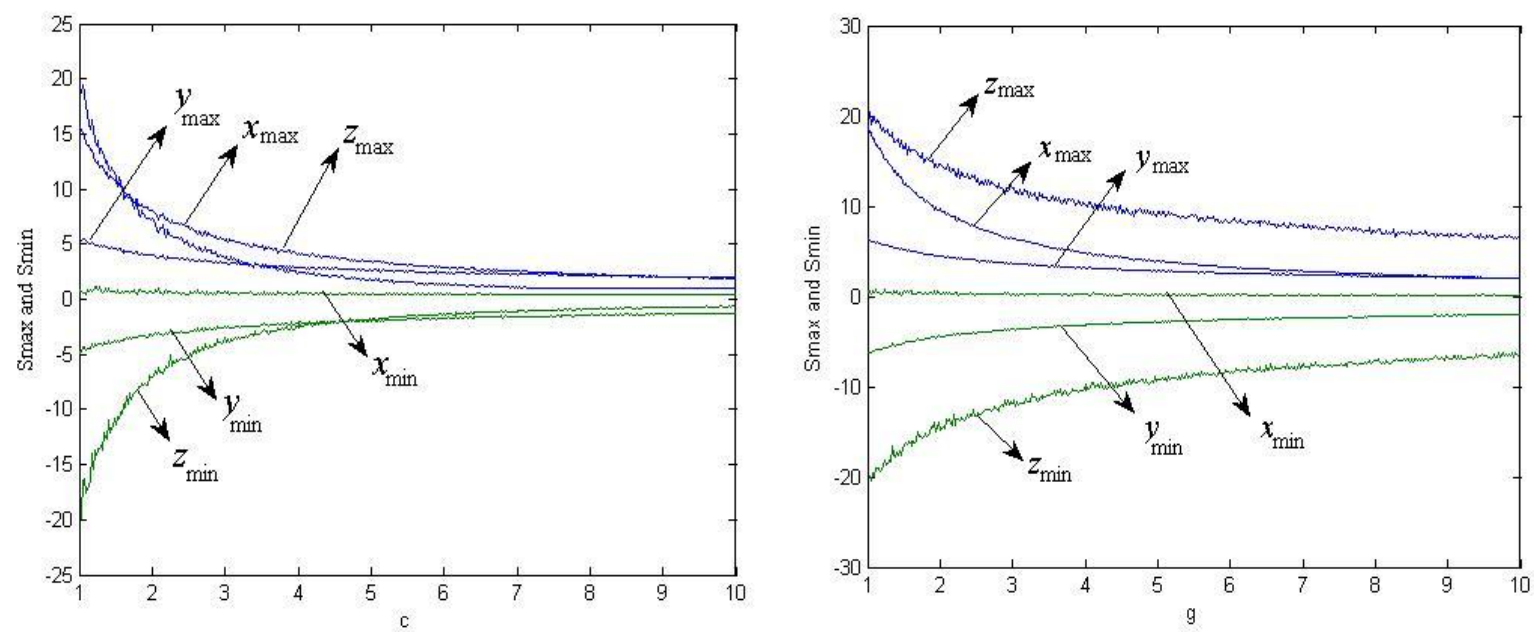

Fig. 5. Signal amplitude with parameters increasing (a) maximum (minimum) values of signals $x, y, z$ versus $c \in\left[\begin{array}{ll}1 & 10\end{array}\right]$ (b) maximum (minimum) values of signals $x, y, z$ versus $g \in\left[\begin{array}{ll}1 & 10\end{array}\right]$.

when the signal $y$ and the parameters $c$ and $g$ anti-changed in system (1), the system equations are not affected, namely $f(x, y, z, a, b, c, d, g)=f(x,-y, z, a, b,-c, d,-g)$, so phase inverted of the parameters $c$ and $g$ with respect to signal $y$ is shown in Fig. 6 . 

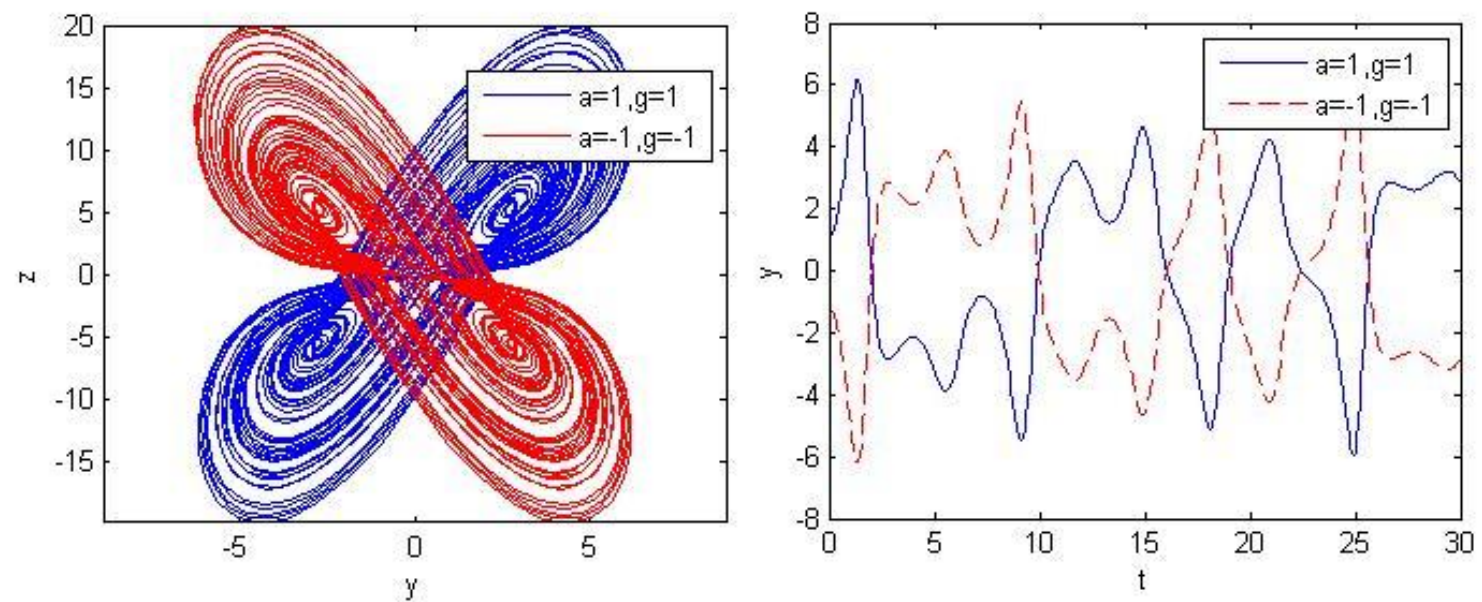

Fig. 6. Phase inversion control (a) $y$-z plane (b) signal $y$.

Fig. 6(a) clearly shows the $y$-z phase diagram in the case of signal $y(t)$ in the initial $(1,1,1)$ and $(1,-1,1)$, respectively, which implies the phase diagram on the $y$ axis symmetry, and Fig. 6 (b) shows the polarity of signal $y$ changed with $a$ and $g$. During the inverting operation, the initial value polarity of $y$ should be changed too, otherwise force role of the initial value will result in the phase inverted failed.

\section{Single Controller Synchronization}

With the in-depth study of chaos synchronization, more and more synchronized methods has been applied to chaotic synchronization, such as active feedback, adaptive, sliding mode, backstepping, and so on. To the best of our knowledge, in the research area of chaotic systems with constant Lyapunov spectrum, this is first to realize the switch synchronization with single controllers via multiple methods. But during the majority process of synchronization, multiple synchronization controllers will make cost increased of every possible industrial areas of application, and the engineering implementation become more complex and difficult in the future, so the development of chaotic synchronization controller will toward the fewer number and simpler structure direction.

Due to the particularity of the structure of system(1), a variety of methods of single controller synchronization can be achieved, and synchronous switching circuit can be realized by using the synchronous switching toolbox.

Consider the drive system

$$
\begin{aligned}
& \dot{x}_{1}=-a\left(x_{1}-y_{1}^{2}\right) \\
& \dot{y}_{1}=-c z_{1}+b y_{1} \\
& \dot{z}_{1}=-d z_{1}+g x_{1} y_{1}
\end{aligned}
$$

The response system

$$
\begin{aligned}
& \dot{x}_{2}=-a\left(x_{2}-y_{2}^{2}\right)+u_{1} \\
& \dot{y}_{2}=-c z_{2}+b y_{2}+u_{2} \\
& \dot{z}_{2}=-d z_{2}+g x_{2} y_{2}+u_{3}
\end{aligned}
$$

where $u_{i}(i=1,2,3)$ are control functions to be determined for achieving synchronization between systems (6) and (7). By taking $e_{1}=x_{2}-x_{1}, e_{2}=y_{2}-y_{1}, e_{3}=z_{2}-z_{1}$, so the error dynamical system is described as 


$$
\begin{aligned}
& \dot{e}_{1}=-a\left(e_{1}-e_{2}^{2}-2 y_{1} e_{2}\right)+u_{1} \\
& \dot{e}_{2}=-c e_{3}+b e_{2}+u_{2} \\
& \dot{e}_{3}=-d e_{3}+g\left(e_{1} e_{2}+y_{1} e_{1}+x_{1} e_{2}\right)+u_{3}
\end{aligned}
$$

When the designed controllers make error system(8) asymptotic stability that implies systems (6) and (7) achieve synchronization.

\subsection{Active Feedback Synchronization}

Theorem 2. If the controllers are designed as $u_{2}=-a \lambda_{1} e_{1} e_{2}+c e_{3}-g e_{1} e_{3}-g x_{1} e_{3}-K_{1} e_{2}, u_{1}=u_{3}=0$. With $\lambda_{1}>0, K_{1}>0, a, c$ and $g$ are parameters of system (1), the controlled system (8) is globally asymptotic stability. That is to say systems (6) and (7) will achieve synchronized under any initial values.

Proof. The following positive Lyapunov function is described

$$
V_{1}=\frac{1}{2} \lambda_{1} e_{1}^{2}+\frac{1}{2} e_{2}^{2}+\frac{1}{2} e_{3}^{2}
$$

where the constant $\lambda_{1}>0$ and will be determined later. Taking the time derivative of (9) along system (8)

$$
\begin{aligned}
\dot{V}_{1} & =\lambda_{1} \dot{e}_{1} e_{1}+\dot{e}_{2} e_{2}+\dot{e}_{3} e_{3} \\
& =-a \lambda_{1}\left(e_{1}^{2}-e_{1} e_{2}^{2}-2 y_{1} e_{2} e_{1}\right)-c e_{2} e_{3}+b e_{2}^{2} \\
& +u_{2}-d e_{3}^{2}+g\left(e_{1} e_{2} e_{3}+y_{1} e_{1} e_{3}+x_{1} e_{2} e_{3}\right) \\
& \leq-a \lambda_{1} e_{1}^{2}+2 \lambda_{1}|a|\left|y_{1}\right| e_{1} e_{2}+\left(b-K_{1}\right) e_{2}^{2}-d e_{3}^{2}+|g|\left|y_{1}\right| e_{1} e_{3} \\
& \leq-E_{1} P_{1} E_{1}
\end{aligned}
$$

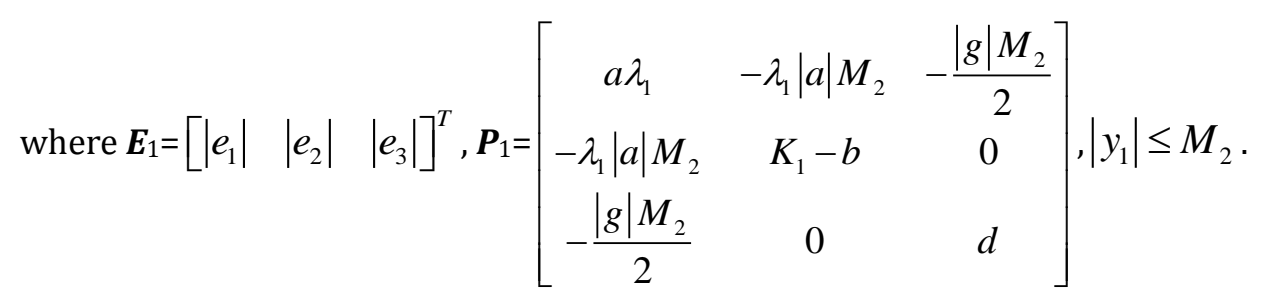

Obviously when $K_{1}>\max \left\{\min _{\lambda_{1}}\left(a \lambda_{1} M_{2}^{2}+b\right), \min _{\lambda_{1}}\left(\frac{4 d a^{2} \lambda_{1}^{2} M_{2}^{2}}{4 a d \lambda_{1}-g^{2} M_{2}^{2}}+b\right)\right\}$, and $\lambda_{1}>\frac{g^{2} M_{2}^{2}}{4 a d}$, matrix $\boldsymbol{P}$ is a positive definite matrix, so $\dot{V}_{1} \leq 0$. System(8) is asymptotically stable, that is to say systems (6) and (7) can achieve synchronization with single controller. This completes the proof of Theorem 2 .

Remark 2: In [18] three controllers are used to synchronize the system. However, according to the active synchronization above, the system in [18] also can be synchronized with single controller.

\subsection{Adaptive Synchronization}

System (6) is referred as the drive system, which drives a similar response system (11) with system parameters uncertain

$$
\begin{aligned}
& \dot{x}_{2}=-\hat{a}\left(x_{2}-y_{2}^{2}\right)+u_{1} \\
& \dot{y}_{2}=-\hat{c} z_{2}+\hat{b} y_{2}+u_{2} \\
& \dot{z}_{2}=-\hat{d} z_{2}+\hat{g} x_{2} y_{2}+u_{3}
\end{aligned}
$$

where $\hat{a}, \hat{b}, \hat{c}, \hat{d}$ are parameters to be determined later, $u_{\mathrm{i}}(i=1,2,3)$ are control functions to realize 
synchronization between systems (6) and (11). Synchronous error are defined as $e_{1}=x_{2}-x_{1}, e_{2}=y_{2}-y_{1}, e_{3}=z_{2}-z_{1}$, the error dynamic system is given by

$$
\begin{aligned}
& \dot{e}_{1}=-a\left(e_{1}-e_{2}^{2}-2 y_{1} e_{2}\right)-e_{a}\left(e_{1}-e_{2}^{2}-y_{1}^{2}-2 y_{1} e_{2}+x_{1}\right)+u_{1} \\
& \dot{e}_{2}=-c e_{3}+b e_{2}-e_{c}\left(e_{3}+z_{1}\right)+e_{b}\left(e_{2}+y_{1}\right)+u_{2} \\
& \dot{e}_{3}=-d e_{3}+g\left(e_{1} e_{2}+y_{1} e_{1}+x_{1} e_{2}\right)-e_{d}\left(e_{3}+z_{1}\right)+e_{g}\left(e_{1} e_{2}+y_{1} e_{1}+x_{1} e_{2}+x_{1} y_{1}\right)+u_{3}
\end{aligned}
$$

where $e_{a}=\hat{a}-a, e_{b}=\hat{b}-b, e_{c}=\hat{c}-c, e_{d}=\hat{d}-d$ 。

Designing synchronous controllers as

$$
u_{2}=-a\left(e_{1} e_{2}+2 y_{1} e_{1}\right)-g \lambda_{2} e_{1} e_{3}-k_{2} e_{2}, u_{1}=u_{3}=0
$$

$k_{2}$ is the feedback gain to be estimated later, and its updated law is

$$
\dot{k}_{2}=\gamma_{1} e_{2}^{2}
$$

$\gamma_{1}$ is a positive constant.

The system parameter updated laws

$$
\begin{aligned}
& \dot{e}_{a}=-\gamma_{2} e_{a}+e_{1}^{2}-e_{2}^{2} e_{1}-2 y_{1} e_{1} e_{2}-y_{1}^{2} e_{1}+x_{1} e_{1} \\
& \dot{e}_{b}=-\gamma_{3} e_{b}-e_{2}^{2}-y_{1} e_{2} \\
& \dot{e}_{c}=-\gamma_{4} e_{c}+e_{2} e_{3}+z_{1} e_{2} \\
& \dot{e}_{d}=-\gamma_{5} e_{d}+e_{3}^{2}+z_{1} e_{3} \\
& \dot{e}_{g}=-\gamma_{6} e_{g}-\lambda_{2}\left(e_{1} e_{2} e_{3}+y_{1} e_{1} e_{3}+x_{1} e_{2} e_{3}+x_{1} y_{1} e_{3}\right)
\end{aligned}
$$

where $\gamma_{i}>0(\mathrm{i}=2,3,4,5,6)$.

Theorem 3. Systems (6) and (11) can achieve synchronization with specified initial values under the action of controllers (13) and parameter updated laws (15).

Proof. The positive Lyapunov function is chosen as

$$
V_{2}=\frac{1}{2} e_{1}^{2}+\frac{1}{2} e_{2}^{2}+\frac{1}{2} \lambda_{2} e_{3}^{2}+\frac{1}{2}\left(e_{a}^{2}+e_{b}^{2}+e_{c}^{2}+e_{d}^{2}+e_{g}^{2}\right)+\frac{\left(k_{2}-K_{2}\right)^{2}}{2 \gamma_{1}} \geq 0
$$

where $K_{2}$ is a positive constant. Taking the time derivative of (16) along system(12), it yields

$$
\begin{aligned}
\dot{V} & =e_{1} \dot{e}_{1}+e_{2} \dot{e}_{2}+\lambda_{2} e_{3} \dot{e}_{3}+e_{a} \dot{e}_{a}+e_{b} \dot{e}_{b}+e_{c} \dot{e}_{c}+e_{d} \dot{e}_{d}+e_{g} \dot{e}_{g}+\frac{\left(k_{2}-K_{2}\right) \dot{\tilde{k}}}{\gamma_{1}} \\
& =-a e_{1}^{2}-c e_{2} e_{3}+b e_{2}^{3}-d \lambda_{2} e_{3}^{2}+g \lambda_{2}\left(y_{1} e_{1} e_{3}+x_{1} e_{2} e_{3}\right)-K e_{2}^{2} \\
& -\gamma_{2} e_{a}^{2}-\gamma_{3} e_{b}^{2}-\gamma_{4} e_{c}^{2}-\gamma_{5} e_{d}^{2}-\gamma_{6} e_{g}^{2} \\
& \leq-E_{2}^{T} P_{2} E_{2}^{T}-E_{q}^{T} Q_{2} E_{q}
\end{aligned}
$$

where $E_{2}=\left[\left|e_{1}\right| \quad\left|e_{2}\right| \quad\left|e_{3}\right|\right]^{T}, E_{q}=\left[\left|e_{a}\right|\left|e_{b}\right| \quad\left|e_{c}\right| \quad\left|e_{d}\right| \mid e_{g}\right]^{T}, Q_{2}=\operatorname{diag}\left(\gamma_{2}, \gamma_{3}, \gamma_{4}, \gamma_{5}, \gamma_{6}\right)$, 


$$
P_{2}=\left[\begin{array}{ccc}
a & 0 & -\frac{\lambda_{2}|g| M_{2}}{2} \\
0 & K_{2}-b & \frac{|c|-|g| \lambda_{2} M_{1}}{2} \\
-\frac{\lambda_{2}|g| M_{2}}{2} & \frac{|c|-|g| \lambda_{2} M_{1}}{2} & d \lambda_{2}
\end{array}\right],\left|x_{1}\right| \leq M_{1},\left|y_{1}\right| \leq M_{2} .
$$

And $K_{2}, \lambda_{2}$ are held

$$
K_{2}>\frac{a d\left(c-g \lambda_{2} M_{1}\right)^{2}}{4 a d \lambda_{2}-g^{2} \lambda_{2}^{2} M_{2}^{2}}, \lambda_{2}<\frac{4 a d}{g^{2} M_{2}^{2}}
$$

Now the matrix $P_{2}$ is positive definite. Obviously, the error system (12) is asymptotically stable, so the systems (6) and (11) can achieve synchronization under the action of controller(13) and adaptive law of parameters (15). This completes the proof of Theorem 3.

Remark 3: The classical system like Lorenz system and lü system can be synchronized via adaptive method with single controller. Do not need to construct multiple controllers like the literature [20].

\subsection{Backstepping Synchronization}

In order to realize synchronization with backstepping method, which is equivalent to control the error system (8). When the system (8) is controlled to zero, then the synchronization between systems (6) and (7) can be achieved.

Step 1:

Consider a positive Lyapunov function $V_{S 1}=\frac{1}{2} e_{1}^{2}$.Its derivative is given by

$$
\dot{V}_{S 1}=e_{1} \dot{e}_{1}=e_{1}\left[-a\left(e_{1}-e_{2}^{2}-2 y_{1} e_{2}\right)+u_{1}\right]
$$

When $u_{1}=0$, and define artificial function $\alpha_{1}\left(e_{1}\right)=0$, then $\dot{V}_{S 1}=-a e_{1}^{2} \leq 0$

Let $\omega_{2}=e_{2}-\alpha_{1}\left(e_{1}\right)=e_{2}$

Step 2:

Define $V_{S 2}=\frac{1}{2} e_{1}^{2}+\frac{1}{2} \omega_{2}^{2}$. Its derivative is given by

$$
\dot{V}_{S 2}=\dot{V}_{S 1}+\omega_{2} \dot{\omega}_{2}=-a e_{1}^{2}+\omega_{2}\left(-c e_{3}+b \omega_{2}+u_{2}\right)
$$

When $u_{2}=0, \alpha_{2}\left(e_{1}, \omega_{2}\right)=k \omega_{2}$, so $\dot{V}_{S 2}=-a e_{1}^{2}+\left(b-c k_{3}\right) \omega_{2}^{2}$

where $k_{3}>\frac{b}{c}$, then $\dot{V}_{s 2} \leq 0$

Define $\omega_{3}=e_{3}-\alpha_{2}\left(e_{1}, \omega_{2}\right)=e_{3}-k_{3} \omega_{2}$.

Step 3:

Choose Lyapunov function $V_{S 3}=\frac{1}{2} e_{1}^{2}+\frac{1}{2} \omega_{2}^{2}+\frac{1}{2} \omega_{3}^{2}$. Its derivative is expressed as

$\dot{V}_{S 3}=\dot{V}_{S 2}+\omega_{3} \dot{\omega}_{3}=-a e_{1}^{2}+\left(b-c k_{3}\right) \omega_{2}^{2}+\omega_{3}\left[-d\left(\omega_{3}+k_{3} \omega_{2}\right)+g\left(e_{1} \omega_{2}+y_{1} e_{1}+x_{1} \omega_{2}\right)+u_{3}-k_{3} \omega_{2}\right]$ 
When $u_{3}=-g\left(e_{1} \omega_{2}+y_{1} e_{1}+x_{1} \omega_{2}\right)+(d+1) k_{3} \omega_{2}$

$$
\dot{V}_{S 3}=-a e_{1}^{2}+\left(b-c k_{3}\right) \omega_{2}^{2}-(d+1) \omega_{3}^{2} \leq 0
$$

According to LaSalle-Yoshizawa theorem it follows $\lim _{t \rightarrow \infty} e_{i}=0 \quad(\mathrm{i}=1,2,3)$, and systems (6) and (7) are globally synchronized.

\subsection{Passsive Synchronization}

Consider the following nonlinear system

$$
\begin{aligned}
& \dot{x}=f(x)+g(x) u \\
& y=h(x)
\end{aligned}
$$

where $x$ is system state variable. $u$ is controller to be determined later. $y$ is the output variable. $f(\mathrm{x})$ and $g(\mathrm{x})$ are smooth vector fields. $h(\mathrm{x})$ is a smooth mapping. The system (17) should hold two conditions such that realize the passive synchronization control [22]

1) The controlled system is minimum phase system;

2) For the controlled system if exists a real value constant $\beta$ such that for any $t \geq 0$, the (24) holds.

$$
\int_{0}^{t} u^{T}(\tau) y(\tau) d \tau \geq \beta
$$

Or exists $\rho>0$ and a real constant $\beta$ for any $t \geq 0$ such that

$$
\int_{0}^{t} u^{T}(\tau) y(\tau) d \tau+\beta \geq \rho \int_{0}^{t} y^{T}(\tau) y(\tau) d \tau
$$

Theorem 4. System (8) is a minimum phase system.

Proof. Suppose $e_{2}$ as system output, and $Z_{1}=e_{1}, Z_{2}=e_{3}, Y=e_{2}, u_{1}=u_{3}=0$, then the system (11) can be rewritten as

$$
\begin{aligned}
& \dot{Z}_{1}=-a\left(Z_{1}-Y^{2}-2 y_{1} Y\right) \\
& \dot{Z}_{2}=-d Z_{2}+g\left(Z_{1} Y+y_{1} Z_{1}+x_{1} Y\right) \\
& \dot{Y}=-c Z_{2}+b Y+u_{2}
\end{aligned}
$$

System (26) can be described by

$$
\begin{aligned}
& \dot{z}=f_{0}(z)+p(z, y) y \\
& \dot{y}=b(z, y)+a(z, y) u_{2}
\end{aligned}
$$

where

$$
\begin{gathered}
z=\left[\begin{array}{ll}
Z_{1} & Z_{2}
\end{array}\right]^{T}, y=Y \\
f_{0}(z)=\left[\begin{array}{ll}
-a Z_{1} & -d Z_{2}+g y_{1} Z_{1}
\end{array}\right]^{T}, \\
p(z, y)=\left[\begin{array}{ll}
a Y+2 a y_{1} & g Z_{1}+g x_{1}
\end{array}\right]^{T} \\
b(z, y)=-c Z_{2}+b Y, \\
a(z, y)=1,
\end{gathered}
$$

Define a storage function candidate 


$$
V(z, y)=W(z)+\frac{y^{2}}{2}
$$

where $W(z)$ is the positive Lyapunov function for $f_{0}(z)$, which is designed to make $f_{0}(z)$ globally asymptotically.

Set

$$
W(z)=\frac{1}{2} Z_{1}^{2}+\frac{1}{2} \kappa Z_{2}^{2} \quad W(0)=0, \kappa \text { is a positive constant }
$$

The zero dynamics of system (27) describes the internal dynamics, which is consistent with external constraint $y=0$, i.e.

$$
\dot{z}=f_{0}(z)
$$

The derivative of $W(z)$

$$
\begin{aligned}
& \dot{W}(z)=\frac{\partial W(z)}{\partial z} f_{0}(z) \\
& =\left[\begin{array}{ll}
Z_{1} & \kappa Z_{2}
\end{array}\right]\left[\begin{array}{ll}
-a Z_{1} & -d Z_{2}+g y_{1} Z_{1}
\end{array}\right]^{T} \\
& =-a Z_{1}^{2}-d \kappa Z_{2}^{2}+g \kappa y_{1} Z_{1} Z_{2} \\
& \leq-|z|^{T} P|z| \leq 0 \\
& P=\left[\begin{array}{cc}
a & -\frac{\kappa|g|\left|y_{1}\right|}{2} \\
-\frac{\kappa|g|\left|y_{1}\right|}{2} & d \kappa
\end{array}\right], \quad \kappa<\frac{4 a d}{g^{2} y_{1}^{2}}, \quad \text { due to } \dot{W}(z) \leq 0 \text {,therefore } f_{0}(z) \text { is globally }
\end{aligned}
$$

asymptotically stable, namely error system (19) is in a minimum phase. This completes the proof of Theorem 4.

Theorem 5. System (26) can be equivalent to globally asymptotically stabilized by the controller $u_{2}=-c e_{3}-b e_{2}-e_{1} e_{2}-2 y_{1} e_{1}-\kappa x_{1} e_{3}-k_{3} e_{2}$ in the case of any initial values.

Proof . Derivative of (28) along the trajectory of system (26) is

$$
\begin{aligned}
\dot{V}(z, y) & =\frac{\partial}{\partial} W(z) \dot{z}+y \dot{y} \\
& =\frac{\partial}{\partial} W(z) f_{0}(z)+\frac{\partial}{\partial z} W(z) p(z, y) y+y\left(b(z, y)+a(z, y) u_{2}\right)
\end{aligned}
$$

System (26) is minimum phase, so

$$
\frac{\partial}{\partial} W(z) f_{0}(z) \leq 0
$$

In order to make $\dot{V}(z, y) \leq 0, u_{2}$ should be holds

$$
u_{2}=a^{-1}(z, y)\left[-b(z, y)-\frac{\partial}{\partial z} W(z) p(z, y)-k_{3} y+\eta\right]
$$

where $k>0, \eta$ is an external signal vector. 
Inequality (31) can be rewritten as

$$
\dot{V}(z, y) \leq \eta y-k_{3} y^{2}
$$

Integrating (32) yields

$$
V(z, y)-V\left(z_{0}, y_{0}\right) \leq \int_{0}^{t} \eta(\tau) y(\tau) d \tau-\int_{0}^{t} k_{3} y^{2}(\tau) d \tau
$$

$V\left(z_{0}, y_{0}\right)$ is constant, by taking $\beta=V\left(z_{0}, y_{0}\right)$, inequality (33) can be described by

$$
V(z, y)+\int_{0}^{t} k_{3} y^{2}(\tau) d \tau \leq \int_{0}^{t} \eta(\tau) y(\tau) d \tau+\beta
$$

$V(z, y) \geq 0$, then

$$
\int_{0}^{t} \eta(\tau) y(\tau) d \tau+\beta \geq \int_{0}^{t} k_{3} y^{2}(\tau) d \tau
$$

Which satisfies definition (25). This completes the proof of theorem 5.

Remark 4: It is important to prove characteristic of minimum phase system of the controlled system, however, because of the different system's structures will lead to constructing $W(z)$ function become very complex such as literature [21], [22]. However, in this section appropriate positive definite matrix $P$ is introduced to greatly simplify the structure of $W(z)$ function. Only need to introduce a positive constant $\kappa$ in the $W(z)$ function, when the $\kappa$ satisfies certain conditions, the system can be proved satisfy the minimum phase characteristic. Compared to the literature [21], [22]. the method presented in this paper is more general in the construction of $W(z)$ function.

\section{Numerical Simulation}
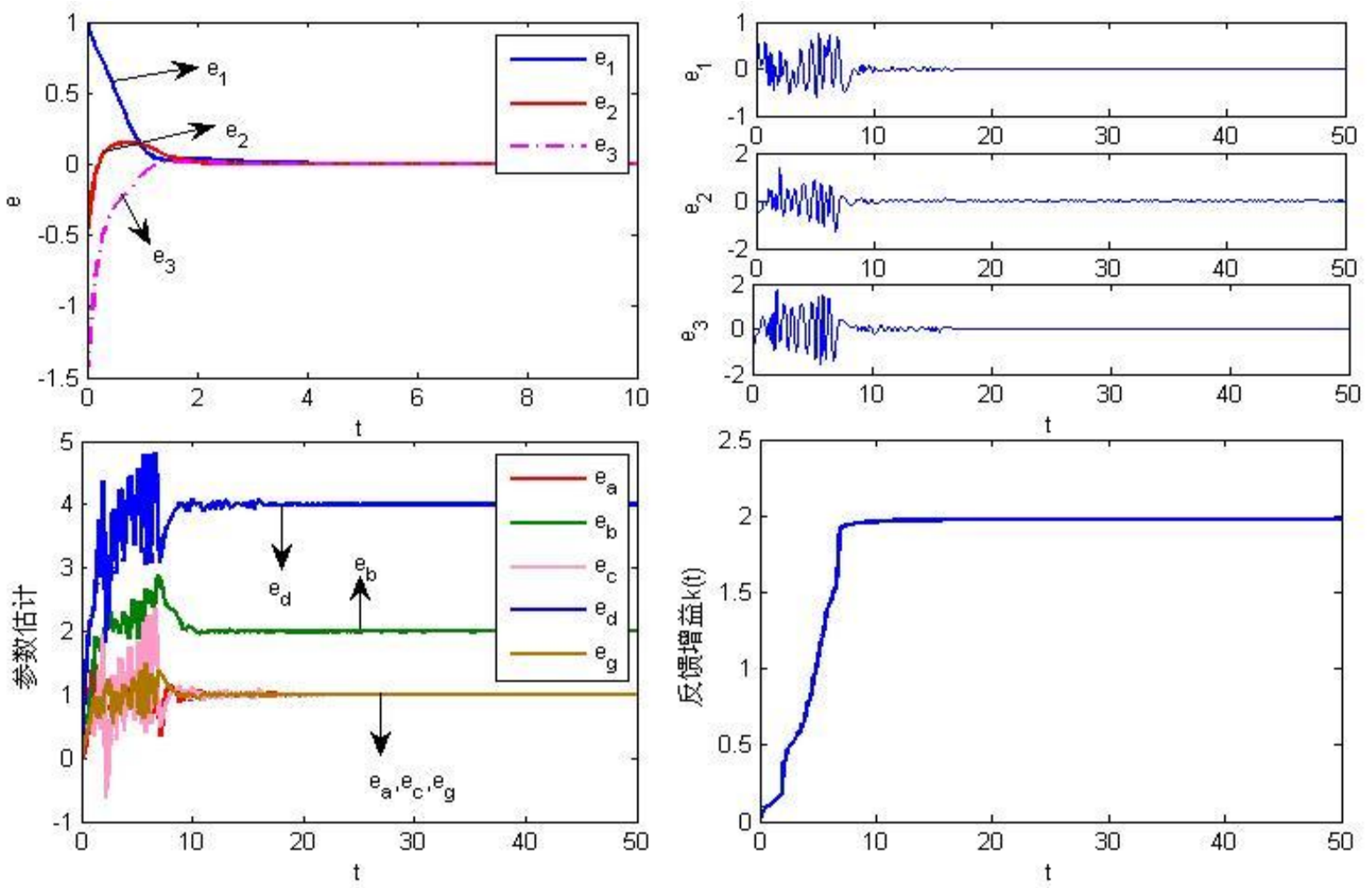

Fig. 8. Synchronization errors and feedback gain varying with time (a) scheme 1 (b) scheme 2 (c) evolution of parameter estimation (d) variation of feedback gain. 
The correctness of the controllers is proved in theory above. The following is to verify the four single controllers by numerical simulation. The ODE45 algorithm is applied to solve the systems based on MATLAB platform. The initial value of drive system and response system are set to $(1,1,1)$ and $(2,0.5,-0.5)$, respectively. Scheme 1: Since the dynamic characteristics of the system has strong robustness to the system parameters $c$ and $g$, so when $a=1, b=2, c=10, d=4, g=1,0<M_{2}<2, \lambda_{1}=0.5, K_{1}=4$. The synchronization error is shown in Fig.7 (a). Scheme 2: when $a=1, b=2, c=1, d=4, g=1,0<M_{1}<20,0 \leq M_{2}<10, \lambda_{2}=0.1$, $e_{a}(0)=e_{b}(0)=e_{c}(0)=e_{d}(0)=e_{g}(0)=0, \gamma_{i}=1,(i=1,2,3, \ldots, 7)$. The synchronization error are shown in Fig.8(a)(b)(c), the changing process of parameters of response system are shown in Fig.8(d), adaptive feedback gain variation in Figure 8 (e). Scheme 3: when $a=1, b=2, c=1, d=4, g=1, k_{3}=4$. The synchronization error of the system is shown in Fig.9 (a). Scheme 4: $a=1, b=2, c=1, d=4, g=1, \kappa=0.01$, $k_{3}=2$. The synchronization error of the system is shown in Fig. 9 (b).
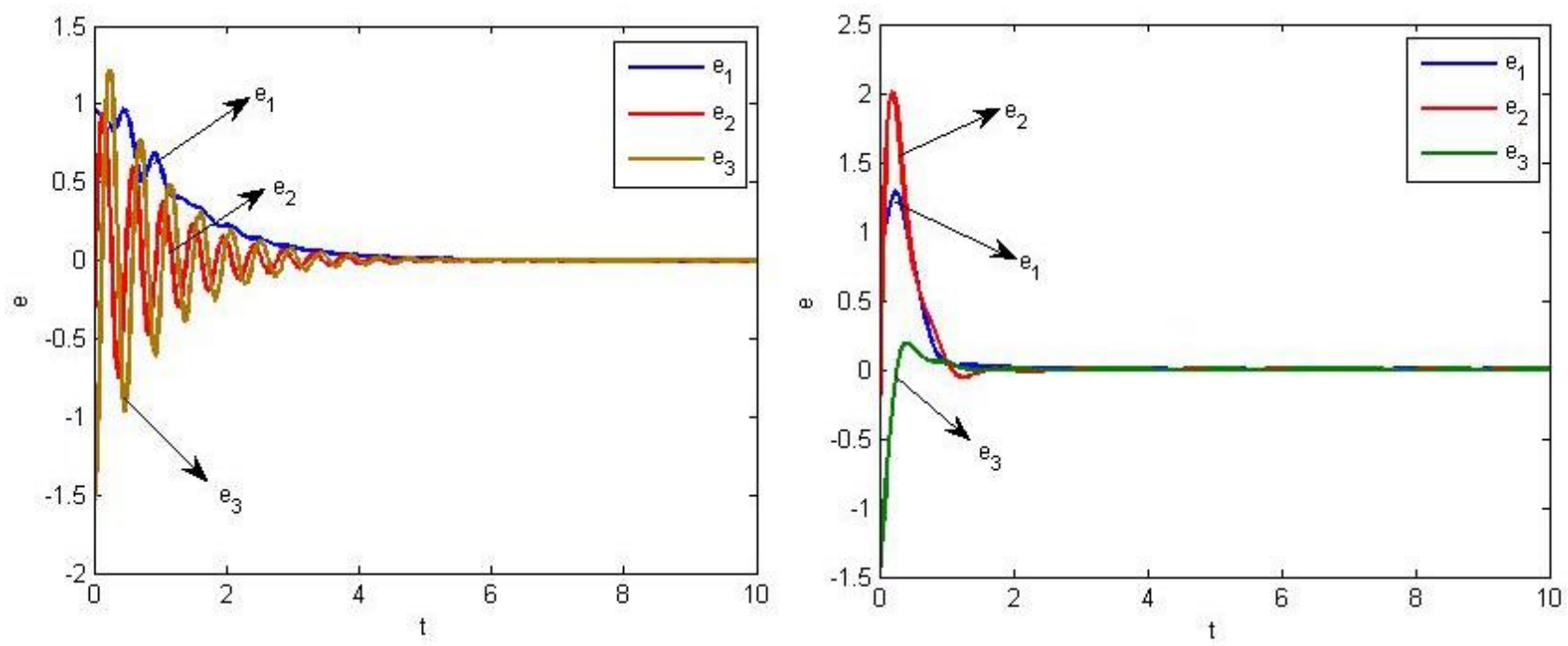

Fig. 9. Synchronization errors (a) scheme 3 (b) scheme 4.

From the error figures above can be seen the error system is gradually stabilized to zero with time, which implies the master-slave systems reached synchronization and further illustrate the correctness of the above schemes. It can be drawn from the synchronization time of the four schemes. The synchronization time of scheme 1 and 4 is short, which can realize rapid synchronization. And the second is scheme 3 . Scheme 2 need for the longest time. Synchronization performance of this new class of chaotic system with constant Lyapunov exponent is good by means of the nonlinear active control, passive control and backstepping approaches. Because of the new systems' synchronization is under the effect of single controller so that it is easy to realized via experiment. Based on the electronic circuit, a synchronous switching toolbox is designed combined with four cases above to further verify the implementation of synchronization schemes.

\section{The Realization of Synchronous Circuit}

Synchronous switching circuit implementation can be realized via the combination of three switches that a single-pole single-throw, single-pole double-throw and a single-pole four-throw switch. The overall design of electronic circuit adopts modular design and electronic components are mainly including operational amplifier LM741H, multiplier AD633, linear resistors and linear capacitance. This paper presents part of the circuit principle diagram and circuit verification results. Part of the circuit diagram of synchronous 
systems and controllers are shown in Fig. 10, 11. The active feedback control is realized as S2 switch to state 1 in the case of switch S3 is in the off state and S1 switch to state 1. Adaptive synchronization control can be achieved with S3 is in off state, S1 switch to the state 2, S2 action to state 2. When S1 switch to the state 1, S2 is in off state, backstepping synchronous control can be reached and with S3 is in closed state. The last passive synchronous control can be achieved with S3 disconnected, S1 switch to state 1, S2 switch to state 3. The synchronization results for four schemes are shown in Fig.12, which shows the phase diagram of synchronization signals in the shape of line segment of $45^{\circ}$, that implies the new system achieved good self-synchronization.
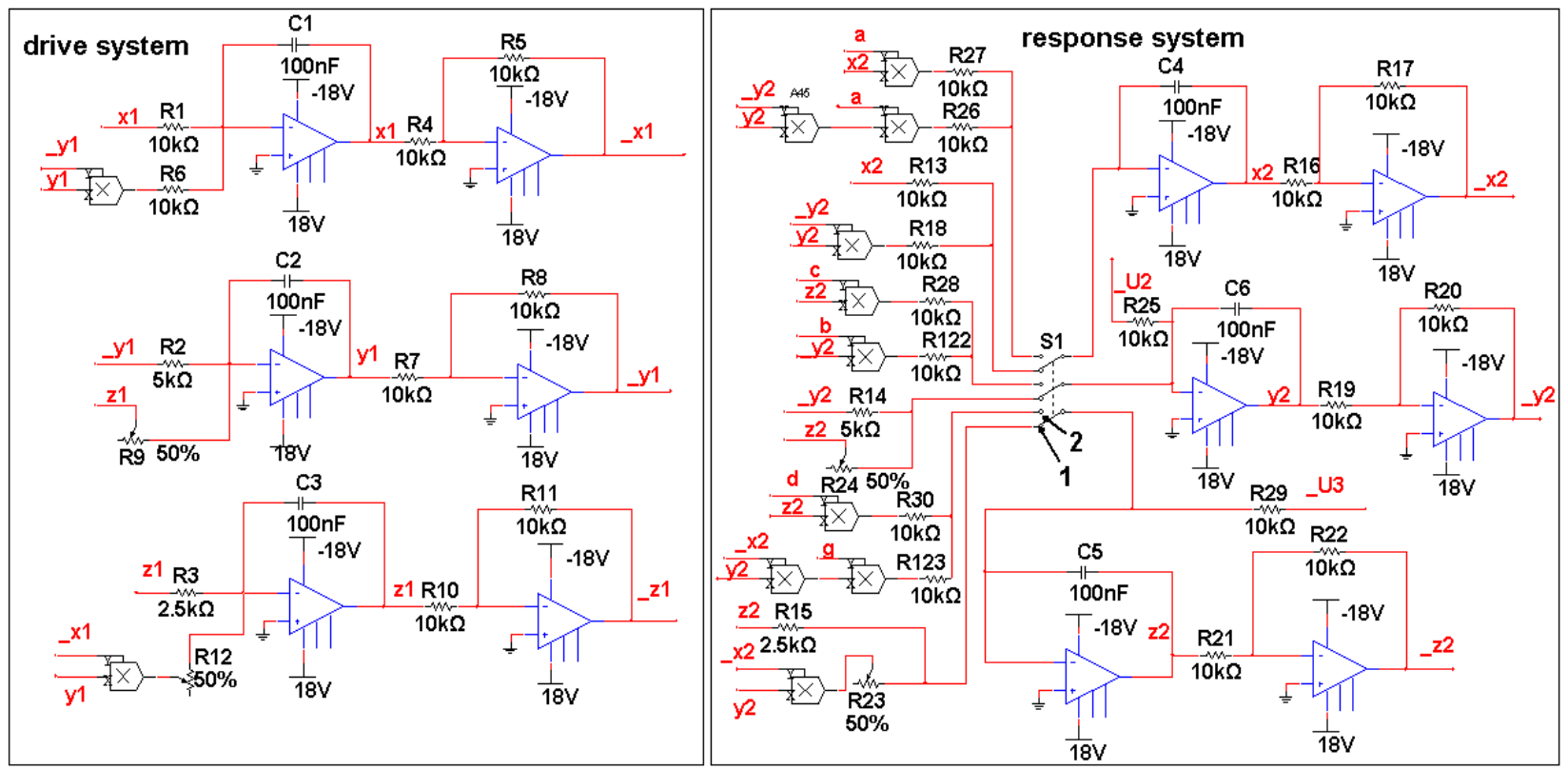

Fig. 10. Circuit implementation of the master system and response system.

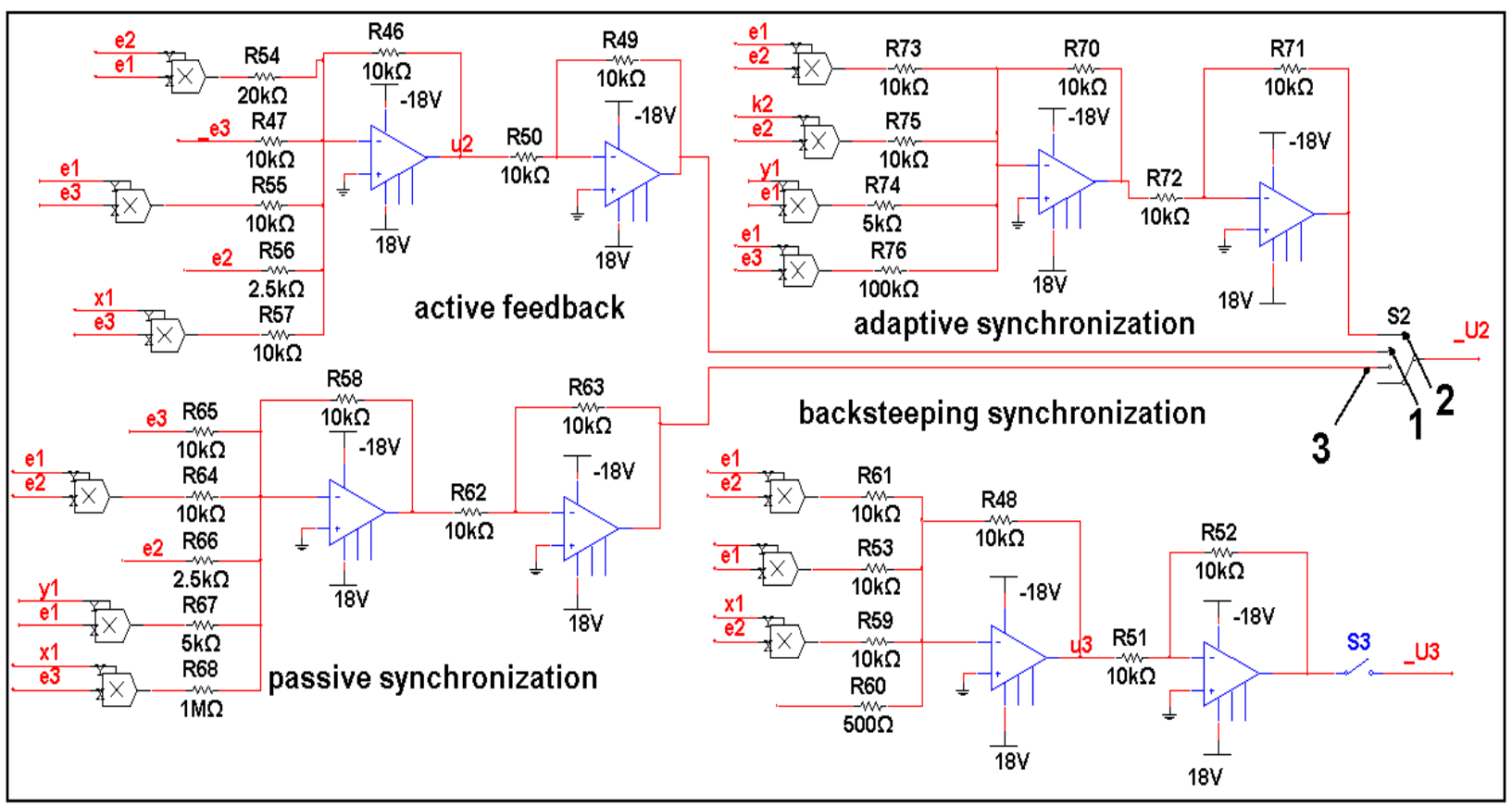

Fig. 11. Circuit implementation of the synchronous switching toolbox. 


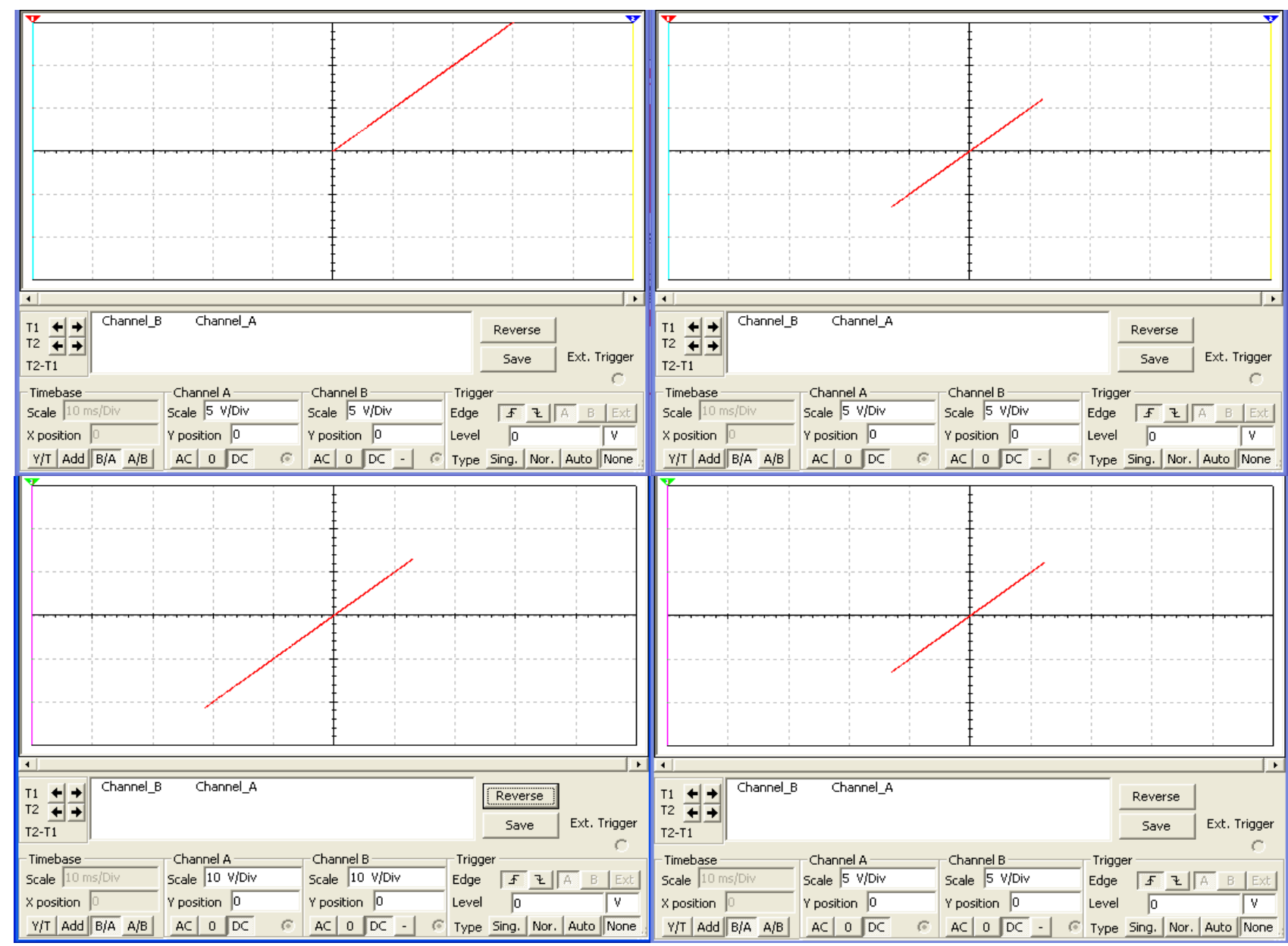

Fig. 12. The synchronization results (a) active feedback control $x_{1}-x_{2}(\mathrm{~b})$ adaptive synchronization control $y_{1}-y_{2}(\mathrm{c})$ backstepping control $z_{1}-z_{2}(\mathrm{~d})$ passive control $y_{1}-y_{2}$.

\section{Conclusion}

This paper presents a new 3D continuous autonomous system with constant Lyapunov exponent spectrum. The chaotic characteristics of the new system are investigated by means of Poincar section, Lyapunov exponents, power spectrum. The relationship between the dynamic characteristics of the system and system parameters with constant Lyapunov exponent spectrum is also investigated in detail, that is verified there exists the global nonlinear amplitude and phase inversion. Based on the single controller the new system is synchronized by using active feedback, adaptive method, backstepping and passive control, respectively, which is helpful for engineering application in the future. The correctness of controllers is proved via Lyapunov stability theory and numerical simulation. In view of the above methods, the switching synchronization toolbox is designed for realizing the synchronization of the system by combining with the four methods with three switches.

\section{References}

[1] Lorenz, E. N. (1963). Deterministic nonperiodic flow. Journal of the Atmospheric Sciences, 20(2), 130-141.

[2] Pecora, L. M., \& Carroll, T. L. (1990). Driving systems with chaotic signals. Physics Review Letters, 44(8), 2374-2383.

[3] Pecora, L. M., \& Carroll, T. L. (1990). Synchronization in chaotic systems. Physical review letters, 64(8), 821-824.

[4] Yang, J., Chen, Y., \& Zhu, F. (2014). Singular reduced-order observer-based synchronization for 
uncertain chaotic systems subject to channel disturbance and chaos-based secure communication. Applied Mathematics and Computation, 229, 227-238.

[5] Shrestha, V. L., Ma, Q., Haider, M. R., et al. (2013). A low-power biologically-inspired chaotic oscillator with process and temperature tolerance. Analog Integrated Circuits and Signal Processing, 74(1), 291-296.

[6] Hao, L., \& Min, L. (2014). Statistical tests and chaotic synchronization based pseudorandom number generator for string bit sequences with application to image encryption. The European Physical Journal Special Topics, 1-19.

[7] Guan, Z. H., \& Lai, Q., Chi, M., et al. (2014). Analysis of a new three-dimensional system with multiple chaotic attractors. Nonlinear Dynamics, 75(1-2), 331-343.

[8] Zhou, P., \& Huang, K. (2014). A new 4-D non-equilibrium fractional-order chaotic system and its circuit implementation. Communications in Nonlinear Science and Numerical Simulation, 19(6), 2005-2011.

[9] Chen, D., Wu, C., Iu, H. H. C., et al. (2013). Circuit simulation for synchronization of a fractional-order and integer-order chaotic system. Nonlinear Dynamics, 73(3), 1671-1686.

[10] Kingni, S. T., Jafari, S., Simo, H., et al. (2014). Three-dimensional chaotic autonomous system with only one stable equilibrium: Analysis, circuit design, parameter estimation, control, synchronization and its fractional-order form. The European Physical Journal Plus, 129(5), 1-16.

[11] Chang-Chun, S., En-Liang, Z., \& Qi-Cheng, X. (2014). Generation of a novel spherical chaotic attractor from a new three-dimensional system. Chinese Physics B, 23(5).

[12] Cang, S., Qi, G., \& Chen, Z. (2010). A four-wing hyper-chaotic attractor and transient chaos generated from a new 4-D quadratic autonomous system. Nonlinear Dynamics, 59(3), 515-527.

[13] Guan, Z. H., Lai, Q., Chi, M., et al. (2014). Analysis of a new three-dimensional system with multiple chaotic attractors. Nonlinear Dynamics, 75(1-2), 331-343.

[14] Li, C. B., \& Hu, W. (2010). Synchronization methods and properties of the mi proved chaotic system with constant Lyapunov exponent spectrum. Acta Phys. Sin, 59(2), 801-815

[15] Li, C. B., Xu, K. S., \& Hu, W. (2011). Sprott system locked on chaos with constant Lyapunov exponent spectrum and its anti-synchronization. Acta Phys. Sin, 60(12).

[16] Li, C. B., Wang, H. K., \& Chen, S. (2010). A novelchaotic attractorwith constant Lyapunov exponent spectrum and its circuit mi plementation. Acta Phys. Sin, 59(2), 783-791

[17] Zhou, X. Y. (2011). A chaotic system with invariable Lyapunov exponent and its circuit simulation. Acta Phys. Sin, 60(10).

[18] Das, S., Srivastava, M., \& Leung, A. Y. T. (2013). Hybrid phase synchronization between identical and nonidentical three-dimensional chaotic systems using the active control method. Nonlinear Dynamics, 73(4), 2261-2272.

[19] Sun, J., Shen, Y., Wang, X., et al. (2014). Finite-time combination-combination synchronization of four different chaotic systems with unknown parameters via sliding mode control. Nonlinear Dynamics, 76(1), 383-397

[20] Adloo, H., \& Roopaei, M. (2011). Review article on adaptive synchronization of chaotic systems with unknown parameters. Nonlinear Dynamics, 65(1-2), 141-159.

[21] Fa-Qiang, W., \& Chong-Xin, L. (2006). Synchronization of hyperchaotic Lorenz system based on passive control. Chinese Physics, 15(9), 1971.

[22] Qun-Jiao, Z., \& Jun-An, L. (2008). Passive control and synchronization of hyperchaotic Chen system. Chinese Physics B, 17(2), 492.

[23] Wei, D. Q., \& Luo, X. S. (2007). Passivity-based adaptive control of chaotic oscillations in power system. Chaos, Solitons and Fractals, 31(3), 665-671. 
[24] Lü, J., \& Chen, G. (2002). A new chaotic attractor coined. International Journal of Bifurcation and Chaos, $12(3), 659-661$.

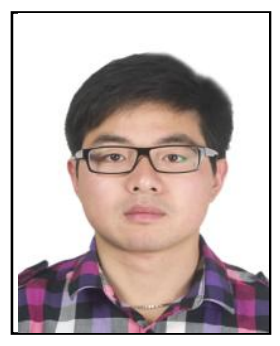

Jian Jiang received his master's degree in software engineering from Shanghai 's Fudan University. And his main research field for enterprise information, synchronization, industrial automation. Since 2010, he has been engaging in enterprise information and industrial automation construction for five years.

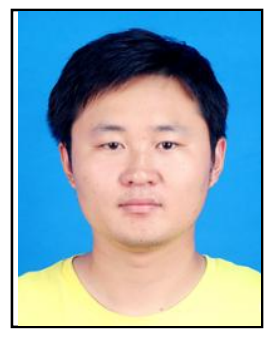

Qing-Qing Wu received his master's degree in system science from Nanjing University of Information Science and Technology, the main research field is synchronization, nonlinear control. 